\title{
A New Sliding Function for Discrete Predictive Sliding Mode Control of Time Delay Systems
}

\author{
Abdennebi Nizar \\ Ben Mansour Houda \\ Nouri Ahmed Said \\ Numerical Control of Industrial Processes, National Engineering School of Gabes, University of Gabes, Gabes 6029, Tunisia
}

\begin{abstract}
The control of time delay systems is still an open area for research. This paper proposes an enhanced model predictive discrete-time sliding mode control with a new sliding function for a linear system with state delay. Firstly, a new sliding function including a present value and a past value of the state, called dynamic surface, is designed by means of linear matrix inequalities (LMIs). Then, using this dynamic function and the rolling optimization method in the predictive control strategy, a discrete predictive sliding mode controller is synthesized. This new strategy is proposed to eliminate the undesirable effect of the delay term in the closed loop system. Also, the designed control strategy is more robust, and has a chattering reduction property and a faster convergence of the system's state. Finally, a numerical example is given to illustrate the effectiveness of the proposed control.
\end{abstract}

Keywords: State time delay systems, discrete sliding mode control, model predictive control, dynamic sliding function, linear matrix inequalities (LMIs), chattering.

\section{Introduction}

Many industrial systems are nonlinear, multivariable, and with parameter uncertainties, external disturbances and time delays. Time delays, as the direct consequences of finite capability of information processing and data transmission among various parts of systems, cannot be neglected or approximated in many cases. These delays can affect the state input or/and output, and they can be constant or time varying, known or unknown, deterministic or stochastic depending on the systems under consideration. Time delays, regardless if they are present in the control or/and the state or/and the output, can affect the system stability and degrade the control performance even in a linear system. In addition, they can produce a decrease in the system phase and can also give rise to a non-rational transfer function of the system ${ }^{[1-13]}$. These complexities of the system make the design of an accurate mathematical model and the development of an adequate control difficult. Then controlling this kind of systems can be a challenging task, especially in the presence of mismatched time varying uncertainties, parameter variations and external disturbances. In recent years, the control problem of time delay systems has received considerable attention and different design approaches have been proposed ${ }^{[1]}$.

Among the various methods developed to control timedelay systems, the sliding mode control (SMC) has been extensively used in both continuous and discrete time ${ }^{[10-18]}$. Sliding mode control design is composed of two steps. In the first step, a custom-made surface must be designed. Whereas on the sliding regime, the plant's dynamics is restricted to the equations of the surface, and is robust to plant uncertainties and external disturbances. In the second step, a feedback control law is designed the system trajectory converges to the sliding surface in a finite time. The system's motion on the sliding surface is called the slid-

\footnotetext{
Manuscript received February 15, 2013; revised May 28, 2013

This work was supported by Ministry of the Higher Education and Scientific Research in Tunisa.
}

ing mode. However, the undesired chattering produced by the high frequency switching of the control may be considered as a problem in implementing the sliding mode control methods for some real applications ${ }^{[16-23]}$.

Model based predictive control (MPC) has been successfully implemented for the dead time process ${ }^{[3,5,6,24]}$. The basic idea of MPC is to calculate a sequence of future control signals in such a way that it can minimize a multistage cost function defined over a prediction horizon. The index to be optimized is between the predictive system output and some predictive reference sequence over the horizon plus a quadratic function measuring control effort ${ }^{[25,26]}$. In order to implement an MPC, a plant's model is used to predict the future plant output. This prediction is based on the past and current values of the input and the output of the plant. The merits of the MPC algorithm include its ability to obtain prediction of the considered signals, to make use of the well known state space model, and to handle imposed hard constraints on the system. Also, it can be used to control a great variety of processes, ranging from those with relatively simple dynamics to those more complex ones, including systems with long dead time, non-minimum phase, switched and unstable ones ${ }^{[3,27]}$. In spite of these advantages, MPC has some limitations. The computational cost can be very high, particularly if a high control horizon is chosen and when constraints are present. Nevertheless the control law is model dependent, a better model is required to guarantee the success of MPC control strategy. Because of the finite horizon, the stability and robustness of the process are difficult to analyze and guarantee, especially when constraints are present.

In order to achieve the advantages of the two techniques of SMC and MPC, a model predictive sliding mode control has been recently proposed. Among the first contribution in this kind of control strategy, we can cite [28-31], which are based on the use of the MPC to choose the coefficient of the discontinuous part of the control law. However, there are a few results reported for predictive sliding mode control for 
state time delay systems. Mansour et al. ${ }^{[32]}$ introduced the prediction of the sliding surface into the control objective function for uncertain systems with external disturbances.

The main idea of this work is to introduce a new sliding surface which depends on the present and past values of the state system. This sliding function was used by [14, $15]$ on controlling the time delay systems with and without time varying uncertainties. So in this paper, we take into account the prediction of this new sliding surface into the control objective function when we synthesize the control law.

This paper is organized as follows. Section 2 describes the basic concepts by giving the system description and preliminaries, the sliding mode control and the model predictive control theories. Sections 3 and 4 describe the synthesis of the predictive sliding mode controller with the classic and the dynamic sliding functions. In Section 5, the advantages of the presented controller are verified by a numerical simulation example. Conclusions and future works are developed in Section 6.

\section{Basic concepts}

\subsection{System description}

Consider the following discrete-time delay system

$$
\left\{\begin{array}{l}
x(k+1)=A x(k)+A_{d} x(k-d)+B u(k) \\
x(k)=\varphi(k), \quad k \in\{-d,-d+1, \cdots, 0\}
\end{array}\right.
$$

where $x \in \mathbf{R}^{n}$ is the state system vector, $u \in \mathbf{R}^{m}$ is the control input, $A, A_{d}$ and $B$ are matrices with appropriate dimensions, $d$ is the constant delay, and $\varphi(k)$ is a vectorvalued initial state function.

For the convenience of the proof and without loss of generality, we can take the following assumptions.

Assumption 1. All system states are available.

Assumption 2. The pair of matrices $\left(A+A_{d}, B\right)$ is controllable.

\section{$2.2 \quad$ Sliding mode control}

In this section, we analyze the sliding mode dynamic. For the discrete-time delay system (1), the classical sliding function is defined by

$$
s(k)=C x(k)
$$

where $C \in \mathbf{R}^{m \times n}$. For system (1), the reaching condition is described as ${ }^{[22,23]}$

$$
|s(k+1)|-|s(k)|<0 .
$$

According to sliding mode function (2), the sliding mode value at time $k+1$ can be obtained as

$$
\begin{aligned}
& s(k+1)=C x(k+1) \\
& s(k+1)=C\left[A x(k)+A_{d} x(k-d)+B u(k)\right] .
\end{aligned}
$$

For the purpose of obtaining some desired performance, such as strong robustness, fast convergence and chattering elimination, we introduce a reaching law to ensure the convergence of the sliding function $s(k)$ to zero.
To ensure a quasi-sliding mode, the sliding mode function must verify the reaching law ${ }^{[17,18,20]}$ :

$$
s(k+1)=(1-q T) s(k)-\varepsilon T \operatorname{sgn}(s(k))
$$

with $0<1-q T<1$ and $0<\varepsilon T<1$, where $T>0$, $\varepsilon>0$ and $q>0$ are the sampling period, reaching rate and approximation rate index. Or

$$
\begin{aligned}
s(k+1)-s(k)= & C x(k+1)-C x(k) \\
s(k+1)-s(k)= & C A x(k)+C A_{d} x(k-d)+ \\
& C B u(k)-C x(k) \\
s(k+1)-s(k)= & -\varepsilon T \operatorname{sgn}(s(k))-q T s(k) .
\end{aligned}
$$

Solving for $u(k)$ gives the discrete sliding mode control law as

$$
\begin{aligned}
u(k)= & (C B)^{-1}\left[-C A x(k)-C A_{d} x(k-d)+\right. \\
& C x(k)-q T s(k)-\varepsilon T \operatorname{sgn}(s(k))] .
\end{aligned}
$$

\subsection{Model predictive control}

MPC has been successfully implemented in many industrial applications, showing good performance ${ }^{[3,24-26]}$.

MPC does not designate a specific control strategy but a range of control methods, which makes an explicit use of a model of the process to obtain the control signal by minimizing an objective function. The ideas appearing in all the predictive control family are basically ${ }^{[26]}$ :

1) The explicit use of a model to predict the process output at future time instants (horizon);

2) The calculation of control sequence minimizing an objective function;

3) At each instant, the horizon is displaced towards the future, which involves the application of the first control signal of the sequence calculated at each step.

The basic idea of MPC is to calculate a sequence of future control signals in such a way that it minimizes a multistage cost function of the form ${ }^{[25,26,31]}$ :

$$
\begin{aligned}
J\left(N^{\prime}, N, M\right)= & \sum_{j=N^{\prime}}^{N} q(j)[w(k+j)-y(k+j / k)]^{2}+ \\
& \sum_{j=1}^{M} \lambda(j)[\Delta u(k+j-1)]^{2}
\end{aligned}
$$

where $y(k+j / k)$ is an optimum $j$-step ahead prediction of the system output on data up to time $k, N^{\prime}$ and $N$ are the minimum and maximum costing horizons, $M$ is the control horizon, $q(j)$ and $\lambda(j)$ are the weighting sequences, and $w(k+j)$ is the future reference trajectory.

The objective of MPC is to compute the future control sequence $u(k), u(k+1), \cdots$ in such a way that the future plant output $y(k+j)$ is driven close to $w(k+j)$ by minimizing the quadratic cost function $J\left(N^{\prime}, N, M\right)$.

\section{Synthesis of discrete predictive slid- ing mode controller with classic slid- ing function}

\subsection{Control law synthesis}

In this section, we consider the discrete predictive sliding mode control (DPSMC) problem for system 1. We take the 
reaching law proposed in $[27,30,33]$ as a reference sliding mode trajectory. It is defined as

$$
\left\{\begin{array}{l}
s_{r}(k+p)=(1-q T) s_{r}(k+p-1) \xi(k) \\
-\varepsilon T \operatorname{sgn}(s(k+p-1)) \psi(k) \\
s_{r}(k)=s(k)
\end{array}\right.
$$

where

$$
\begin{gathered}
\xi(k)=\left\{\begin{array}{cc}
1, & |s(k)|>\eta \\
0, & |s(k)|<\eta
\end{array}\right. \\
\psi(k)= \begin{cases}1, & |s(k)|>\eta \\
\frac{|s(k)|^{2}}{\eta}, & |s(k)|<\eta\end{cases}
\end{gathered}
$$

with $0<1-q T<1,0<\varepsilon T<1$ and $\eta=\frac{T}{1-q T}$.

The objective is to design a sliding mode predictive control. Firstly, we calculate the sliding function (2) at time $k+p$. We have

$$
\begin{aligned}
s(k+p)= & C A^{p} x(k)+\sum_{i=1}^{p} C A_{d}^{i-1} x(k-d+p-i)+ \\
& \sum_{j=1}^{p} C A^{j-1} B u(k+p-j)
\end{aligned}
$$

where $k \in \mathbf{Z}$ and $p \in \mathbf{Z}$.

Then, the predictive sliding mode value of time $k$ on time $k-p$ can be deduced as

$$
\begin{aligned}
s(k / k-p)= & C A^{p} x(k-p)+\sum_{i=1}^{p} C A_{d}^{i-1} x(k-d-i)+ \\
& \sum_{j=1}^{p} C A^{j-1} B u(k-j) .
\end{aligned}
$$

Equality (10) can be described in a vector form as

$$
S_{p}(k+1)=\Gamma X(k)+\Pi X_{d}(k)+\Omega U(k)
$$

where

$$
\begin{aligned}
& S_{p}(k+1)=[s(k+1), s(k+2), \cdots, s(k+N)]^{\mathrm{T}} \\
& X(k)=\left[\begin{array}{c}
x(k) \\
x(k) \\
\cdots \\
x(k)
\end{array}\right]_{N \times 1} \\
& X_{d}(k)=\left[\begin{array}{c}
x(k-d) \\
x(k-d+1) \\
\cdots \\
x(k-d+N-1)
\end{array}\right]_{N \times 1} \\
& U(k)=[u(k), u(k+1), \cdots, u(k+M-1)]^{\mathrm{T}} \\
& \Gamma=\left[(C A)^{\mathrm{T}}\left(C A^{2}\right)^{\mathrm{T}} \cdots\left(C A^{N}\right)^{\mathrm{T}}\right]^{\mathrm{T}} \\
& \Pi=\left[\begin{array}{cccccc}
C A_{d} & 0 & 0 & \ldots & 0 & 0 \\
C A A_{d} & C A_{d} & 0 & \ldots & 0 & 0 \\
C A^{2} A_{d} & C A A_{d} & C A_{d} & \ldots & \ldots & \ldots \\
C A^{3} A_{d} & C A^{2} A_{d} & C A A_{d} & C A_{d} & \ldots & \ldots \\
\cdots & \cdots & \ldots & \cdots & \ldots & \ldots \\
C A^{N-1} A_{d} & C A^{N-2} A_{d} & \ldots & \ldots & \ldots & C A_{d}
\end{array}\right]
\end{aligned}
$$

$$
\Omega=\left[\begin{array}{ccccc}
C B & \ldots & \ldots & \ldots & 0 \\
C A B & \ldots & \ldots & \ldots & 0 \\
\cdots & \ldots & \ldots & \ldots & \ldots \\
C A^{M-1} B & \ldots & \ldots & C A B & C B \\
\ldots & \ldots & \ldots & \ldots & \ldots \\
C A^{N-2} B & \ldots & \ldots & C A^{N-M} B & C A^{N-M-1} B \\
C A^{N-1} B & \ldots & \ldots & C A^{N-M+1} B & C A^{N-M} B
\end{array}\right]
$$

with $N$ being the prediction horizon, $M$ being the control horizon, and the minimum costing horizon $N^{\prime}$ being chosen to be 1 . In practice, to make correction to the future predictive sliding mode value $s(k+p)$, we introduce the error between practical sliding mode value $s(k)$ and the predictive sliding mode value $s(k / k-p)$. Therefore, the output of the sliding mode prediction $\tilde{s}_{p}(k+p)$ is given as

$$
\begin{aligned}
\tilde{s}_{p}(k+p)= & s(k+p)+h_{p} e(k)= \\
& C A^{p} x(k)+\sum_{i=1}^{p} C A_{d}^{i-1} x(k-d+p-i)+ \\
& \sum_{j=1}^{p} C A^{j-1} B u(k+p-j)+h_{p} e(k)
\end{aligned}
$$

where $e(k)=s(k)-s(k / k-p)$ and $h_{p}$ is the correct coefficient.

Rewrite (12) in a vector form:

$$
\tilde{S}_{p}(k+1)=S_{p}(k+1)+H_{p} E(k)
$$

where

$$
\begin{gathered}
\tilde{S}_{p}(k+1)=[\tilde{s}(k+1), \tilde{s}(k+2), \cdots, \tilde{s}(k+N)]^{\mathrm{T}} \\
H_{p}=\operatorname{diag}\left\{h_{1}, h_{2}, \cdots, h_{N}\right\} \\
E(k)=S(k)-S_{m p}(k) \\
S(k)=[s(k), s(k), \cdots, s(k)]_{1 \times N}
\end{gathered}
$$

$$
S_{m p}(k)=[s(k / k-1), s(k / k-2), \cdots, s(k / k-N)]^{\mathrm{T}} .
$$

The following corresponding optimization cost function is defined in as ${ }^{[27,30,31]}$

$$
\begin{aligned}
j_{p}= & \sum_{j=1}^{N} q_{j}\left[\tilde{s}_{p}(k+1)-s_{r}(k+j)\right]^{2}+ \\
& \sum_{l=1}^{M} g_{l}[u(k+l-1)]^{2}
\end{aligned}
$$

where $s_{r}(k+1)$ is the sliding mode reference trajectory, and $q_{j}$ and $g_{l}$ are weight coefficients.

In order to simplify the synthesis of the controller, we consider $q_{j}=1$ and $g_{l}=g$. It follows that the corresponding optimization cost function is given by

$$
\begin{aligned}
j_{p}= & \sum_{j=1}^{N}\left[\tilde{s}_{p}(k+i)-s_{r}(k+j)\right]^{2}+ \\
& \sum_{l=1}^{M} g[u(k+l-1)]^{2} .
\end{aligned}
$$


Rewrite (15) in a vector form:

$$
\begin{aligned}
j_{p}= & \left\|\tilde{S}_{p}(k+1)-S_{r}(k+1)\right\|+\|U(k)\|_{G}^{2}= \\
& U(k)^{\mathrm{T}} G U(k)+\left[\Gamma X(k)+\Pi X_{d}(k)+\right. \\
& \left.\Omega U(k)+H_{p} E(k)-S_{r}(k+1)\right]^{\mathrm{T}} \times \\
& {\left[\Gamma x(k)+\Pi X_{d}(k)+\Omega U(k)+H_{p} E(k)-S_{r}(k+1)\right] . }
\end{aligned}
$$

Optimize (16), i.e., $\frac{\partial j_{p}}{\partial U(k)}=0$. The optimal discrete predictive sliding mode control law can be obtained as

$$
\begin{gathered}
U(k)=-\left(\Omega^{\mathrm{T}} \Omega+G\right)^{-1} \Omega^{\mathrm{T}}\left[\Gamma X(k)+\Pi X_{d}(k)+\right. \\
\left.H_{p} E(k)-S_{r}(k+1)\right] .
\end{gathered}
$$

Because of rolling optimization, only the present control input signal is implemented, and the next control signal $u(k+1)$ will be calculated recursively by the control law.

\subsection{Design of classical sliding surface using LMIs}

Many works were interested in the design of the sliding function using the LMIs for time delay systems in the discrete time ${ }^{[10-15]}$

Let's consider a second order discrete-time delay system described by ${ }^{[12]}$

$$
\left\{\begin{array}{c}
x_{1}(k+1)=A_{11} x_{1}(k)+A_{d 11} x_{1}(k-d)+A_{12} x_{2}(k) \\
x_{2}(k+1)=A_{21} x_{1}(k)+A_{d 21} x_{1}(k-d)+A_{22} x_{2}(k)+ \\
A_{d 22} x_{2}(k-d)+B u(k) \\
x(k)=\varphi(k), \quad \forall k \in\{-d,-d+1, \cdots, 0\} .
\end{array}\right.
$$

where $x_{1}(k) \in \mathbf{R}^{n-m}$ and $x_{2}(k) \in \mathbf{R}^{m}$ are the system states vectors, $u(k) \in \mathbf{R}^{m}$ is the control input, $A_{i j}, A_{d i j}$, and $B$ are constant matrices with appropriate dimensions with $\operatorname{rank}(B)=m, \varphi(k)$ presents the initial conditions and $d$ is the constant time delay. The sliding function is defined by

$$
S(k)=C x(k)=\left[\begin{array}{ll}
C_{1} & I
\end{array}\right] x(k)
$$

where $C=\left[\begin{array}{ll}C_{1} & I\end{array}\right]$ is a vector with the appropriate dimension to be designed.

Since $\left(A+A_{d} ; B\right)$ is controllable, we can find a $C_{1} \in$ $\mathbf{R}^{m \times(n-m)}$ such that $D_{1}=A_{11}-A_{12} C_{1}+A_{d 11}$ is a Hurwitz matrix. In the sliding mode, we have

$$
S(k)=0 \Rightarrow x_{2}(k)=-C_{1} x_{1}(k) \text {. }
$$

Substituting (20) into system (18), we have

$$
\begin{aligned}
x_{1}(k+1)= & A_{11} x_{1}(k)-A_{12} C_{1} x_{1}(k)+A_{d 11} x_{1}(k-d)= \\
& \left(A_{11}-A_{12} C_{1}\right) x_{1}(k)+A_{d 11} x_{1}(k-d) .
\end{aligned}
$$

The following theorem gives a sufficient condition under which the sliding motion is stable.

Theorem 1. Consider the reduced system with constant delay $d$ as shown in (21). The system is asymptotically stable if there exists matrices $P=P^{\mathrm{T}}>0, Q=Q^{\mathrm{T}}>0$ and $w_{1}$ with appropriate dimensions satisfying the following $\operatorname{LMI}^{[9,14]}$.

$$
\left[\begin{array}{ccc}
W-L & * & * \\
0 & -L & * \\
A_{11} L+A_{12} w_{1} & A_{d 11} L & -L
\end{array}\right]<0
$$

where $W=P^{-1} Q P^{-1}, L=P^{-1}, w_{1}=-C_{1} L$ and ${ }^{*}$ denotes the transposed elements in the symmetric positions.

\section{Synthesis of discrete predictive slid- ing mode controller with dynamic sliding function}

\subsection{Control law synthesis}

The same sliding function trajectory reference introduced in (8) is used. We present our main contribution by introducing a new predictive sliding function (dynamic surface) in designing the discrete predictive sliding mode controller for time delay systems in this section.

We define the dynamic sliding function for the discretetime system with state delay (1) as

$$
S(k)=C x(k)+C_{d} x(k-d)
$$

with $C$ and $C_{d}$ being the vectors with the appropriate dimensions to be designed. Then, the new discrete predictive sliding function is given by

$$
\begin{aligned}
s(k+p)= & C A^{p} x(k)+\sum_{i=1}^{p} C A^{i-1} A_{d} x(k-d+p-i)+ \\
& \sum_{j=1}^{p} C A^{j-1} B u(k+p-j)+C_{d} x(k-d+p) .
\end{aligned}
$$

Therefore, the predictive sliding mode value of time $k$ on time $k-p$ can be deduced as

$$
\begin{aligned}
s(k / k-p)= & C A^{p} x(k-p)+\sum_{i=1}^{p} C A^{i-1} A_{d} x(k-d+p-i)+ \\
& \sum_{j=1}^{p} C A^{j-1} B u(k-j)
\end{aligned}
$$

Equality (24) can be described in a vector form as

$$
S(k+1)=\Gamma X(k)+\Pi X_{d}(k)+\Omega U(k)+X_{d d}(k)
$$

where

$$
\begin{gathered}
S_{p}(k+1)=\left[\begin{array}{c}
s(k+1) \\
s(k+2) \\
\cdots \\
s(k+N)
\end{array}\right] \\
s(k+1)=C A x(k)+\sum_{i=1}^{1} C A^{i-1} A_{d} x(k-d+1-i)+ \\
\sum_{j=1}^{1} C A^{j-1} B u(k+1-j)+C_{d} x(k-d+1) \\
\sum_{j=1}^{2} C A^{j-1} B u(k+2-j)+C_{d} x(k-d+2) \\
\cdots \\
s(k+N)=C A^{2} x(k)+\sum_{i=1}^{2} C A^{i-1} A_{d} x(k-d+1-i)+ \\
\sum_{j=1}^{N} C A^{j-1} B u\left(k+\sum_{i=1}^{N} C A^{i-1} A_{d} x(k-d+N-i)+\right.
\end{gathered}
$$




$$
\begin{gathered}
S_{p}(k+1)=\left[\begin{array}{c}
C A \\
C A^{2} \\
\cdots \\
C A^{N}
\end{array}\right] x(k)+\left[\begin{array}{c}
\sum_{j=1}^{1} C A^{j-1} B u(k+1-j) \\
\sum_{j=1}^{2} C A^{j-1} B u(k+2-j) \\
\ldots \\
\sum_{j=1}^{N} C A^{j-1} B u(k+N-j)
\end{array}\right]+ \\
{\left[\begin{array}{c}
\sum_{i=1}^{1} C A^{i-1} A_{d} x(k-d+1-i) \\
\sum_{i=1}^{2} C A^{i-1} A_{d} x(k-d+2-i) \\
\sum_{i=1}^{N} C A^{i-1} A_{d} x(k-d+N-i)
\end{array}\right]+\left[\begin{array}{c}
C_{d} x(k-d+1) \\
C_{d} x(k-d+2) \\
\cdots \\
C_{d} x(k-d+N)
\end{array}\right]}
\end{gathered}
$$

with $N$ being the prediction horizon, $M$ being the control horizon, and the minimum costing horizon $N^{\prime}$ being chosen to be 1 ,

$$
X_{d d}(k)=\left[\begin{array}{c}
C_{d} x(k-d+1) \\
C_{d} x(k-d+2) \\
\cdots \\
C_{d} x(k-d+N)
\end{array}\right]_{N \times 1},
$$

and $\Gamma, \Pi, \Omega, X(k), X_{d}(k)$ and $U(k)$ have the same definitions as in the previous section.

In practice, to make correction to the future predictive sliding mode value $s(k+p)$, we introduce the error between practical sliding mode value $s(k)$ and the predictive sliding mode value $s(k / k-p)$. Therefore, the output of the sliding mode prediction $\tilde{s}_{p}(k+p)$ is given as

$$
\begin{aligned}
& \widetilde{s}(k+p)= \\
& \quad s(k+p)-h_{p} e(k)=C A^{p} x(k)+ \\
& \quad \sum_{i=1}^{p} C A^{i-1} A_{d} x(k-d+p-i) x_{d}(k)+ \\
& \quad \sum_{j=1}^{p} C A^{j-1} B u(k+p-j)+C_{d} x(k-d+p)-h_{p} e(k)
\end{aligned}
$$

where $h_{p}$ is the correct coefficient and the error $e(k)=$ $s(k)-s(k / k-p)$. In a vector form, (26) is obtained as

$$
\widetilde{S}_{p}(k+1)=S_{p}(k+1)+H_{p} E(k)
$$

where

$$
\begin{gathered}
\widetilde{S}_{p}(k+1)=[\widetilde{s}(k+1), \widetilde{s}(k+2), \cdots, \widetilde{s}(k+N)]^{\mathrm{T}} \\
H_{p}=\operatorname{diag}\left\{h_{1}, h_{2}, \cdots, h_{N}\right\} \\
E(k)=S(k)-S_{m p}(k) \\
S(k)=[s(k), s(k), \cdots, s(k)]_{1 \times N} \\
S_{m p}(k)=[s(k / k-1), s(k / k-2), \cdots, s(k / k-N)]^{\mathrm{T}} .
\end{gathered}
$$

The following corresponding optimization cost function is defined as ${ }^{[27,30,31]}$

$$
\begin{aligned}
j_{p}= & \sum_{j=1}^{N} q_{j}\left[\widetilde{s}_{p}(k+1)-s_{r}(k+j)\right]^{2}+ \\
& \sum_{l=1}^{M} g_{l}[u(k+l-1)]^{2}
\end{aligned}
$$

where $s_{r}(k+1)$ is the sliding mode reference trajectory, and $q_{j}$ and $g_{l}$ are weight coefficients. In order to simplify the synthesis of the controller, we consider $q_{j}=1$ and $g_{l}=g$. So, the following corresponding optimization cost function is written as

$$
\begin{aligned}
j_{p}= & \sum_{j=1}^{N}\left[\widetilde{s}_{p}(k+1)-s_{r}(k+j)\right]^{2}+ \\
& \sum_{l=1}^{M} g[u(k+l-1)]^{2}
\end{aligned}
$$

Describe (29) in a vector form

$$
\begin{aligned}
j_{p}= & \left\|\widetilde{S}_{p}(k+1)-S_{r}(k+1)\right\|+\|U(k)\|_{G}^{2}= \\
& U(k)^{\mathrm{T}} G U(k)+\left[\Gamma X(k)+\Pi X_{d}(k)+\Omega U(k)+X_{d d}(k)+\right. \\
& \left.H_{p} E(k)-S_{r}(k+1)\right]^{\mathrm{T}} \times\left[\Gamma X(k)+\Pi X_{d}(k)+\right. \\
& \left.\Omega U(k)+X_{d d}(k)+H_{p} E(k)-S_{r}(k+1)\right] .
\end{aligned}
$$

Optimize (30), i.e., $\frac{\partial j_{p}}{\partial U(k)}=0$. The optimal discrete predictive sliding mode control law can be obtained as

$$
\begin{gathered}
U(k)=-\left(\Omega^{\mathrm{T}} \Omega+G\right)^{-1} \Omega^{\mathrm{T}}\left[\Gamma X(k)+\Pi X_{d}(k)+X_{d d}(k)+\right. \\
\left.H_{p} E(k)-S_{r}(k+1)\right] .
\end{gathered}
$$

Because of rolling optimization, only the present control input signal is implemented, and the next time control signal $u(k+1)$ will be calculated recursively by the control law.

\subsection{Design of dynamic sliding function us- ing LMIs}

We define the dynamic sliding function for the discretetime system with state delay (18) as

$$
S(k)=C_{1} x_{1}(k)+x_{2}(k)+C_{d} x_{1}(k-d)
$$

with $C_{1} \in \mathbf{R}^{m \times(n-m)}$ and $C_{d} \in \mathbf{R}^{m \times(n-m)}$ being the vectors with appropriate dimensions to be chosen. Since $\left(A+A_{d} ; B\right)$ is controllable, we can find $C_{1}$ and $C_{d}$ such that $D_{2}=A_{11}-A_{12} C_{1}+A_{d 11}-A_{12} C_{d}$ is a Hurwitz matrix. In the sliding mode, we have

$$
\begin{aligned}
& \qquad \begin{array}{l}
S(k)=0 \Rightarrow x_{2}(k)=-C_{1} x_{1}(k)-C_{d} x_{1}(k-d) \\
x_{1}(k+1)=A_{11} x_{1}(k)-A_{12} C_{1} x_{1}(k)- \\
A_{12} C_{d} x_{1}(k-d)+A_{d 11} x_{1}(k-d)= \\
\left(A_{11}-A_{12} C\right) x_{1}(k)+\left(A_{d 11}-A_{12} C_{d}\right) x_{1}(k-d)
\end{array} \\
& x_{1}(k+1)=\bar{A}_{1} x_{1}(k)+\bar{A}_{d 1} x_{1}(k-d) \\
& \text { with } \bar{A}_{1}=A_{11}-A_{12} C_{1} \text { and } \bar{A}_{d 1}=A_{d 11}-A_{12} C_{d} .
\end{aligned}
$$


Theorem 2. Consider the reduced system (34). Given the positive integer $d$, the system is asymptotically stable if there exist matrices $P=P^{\mathrm{T}}>0, Q=Q^{\mathrm{T}}>0, w_{1}$ and $w_{2}$ with appropriate dimensions satisfying the following $\mathrm{LMI}^{[14,15]}$ :

$$
\left[\begin{array}{ccc}
W-L & * & * \\
0 & -L & * \\
A_{d 11} L+A_{12} w_{1} & A_{d 11} L+A_{12} w_{2} & -L
\end{array}\right]<0
$$

where $W=P^{-1} Q P^{-1}, L=P^{-1}, w_{1}=-C_{1} L$, and $w_{2}=$ $-C_{d} L$. Given this theorem, one can fix these coefficients of this surface optimally based on stability analysis with the candidate Lyapunov function.

\section{Simulation results}

In order to show the considerable contribution in the performance of the proposed control scheme, we consider a discrete-time system with state time delay described by the following representations

$$
\left\{\begin{array}{l}
x(k+1)=A x(k)+A_{d} x(k-d)+B u(k) \\
x(k)=\varphi(k), \quad k=\{-d,-d+1, \cdots, 0\}
\end{array}\right.
$$

where

$$
A=\left[\begin{array}{cc}
1.2 & 0.1 \\
1 & 0.6
\end{array}\right], \quad A_{d}=\left[\begin{array}{cc}
-0.2 & 0 \\
1 & -1
\end{array}\right], B=\left[\begin{array}{l}
0 \\
1
\end{array}\right]
$$

The initial states of the system are $\varphi(k)^{\mathrm{T}}=$ $[-0.7 ; 0.5]$ for all $k=\{-d, \cdots, 0\}, d=20$ is the constant time delay. The vectors of the sliding functions $C$ and $C_{d}$ are designed according to Theorems 1 and 2 :

1) Classic function: $S(k)=5 x_{1}(k)+x_{2}(k)$;

2) Dynamic function: $S(k)=15 x_{1}(k)+x_{2}(k)-2 x_{1}(k-d)$.

The parameters of the predictive sliding mode control are:

1) The predictive horizon $N=10$ and the control horizon $M=5$;

2) The correct coefficient matrix $H_{p}=0.001 \times$ $\operatorname{diag}\left\{\begin{array}{llllllllll}1 & 0.8 & 0.6 & 0.5 & 0.3 & 0.2 & 0.2 & 0.4 & 0.1 & 0.05\} ; ;\end{array}\right.$

3) The control weight coefficient $G=0.001 I_{M \times M}$.

According to the system's dynamics, a sampling period $T$ is chosen as $0.01 \mathrm{~s}$. For the discrete reaching law (8), the retained synthesis parameters are $q=100$ and $\varepsilon=5$.

The simulation results of the predictive sliding mode control (PSMC) with the dynamic sliding function are compared with those of the classical (SMC) and the predictive sliding mode control (PSMC) with the classic sliding function. This is shown in Figs. $1-4$. Fig. 2 shows the evolutions of the states $x_{1}(k)$ and $x_{2}(k)$. Fig. 3 presents the evolutions of the control signal $u(k)$. The evolution of the sliding surfaces is presented in Fig. 4.

Figs. 1 and 2 show that with the proposed technique, the closed-loop system has faster convergence with better performance and that the smooth control is allowed to apply to the system considered as shown in Fig. 3. The discrete predictive sliding mode control with the classic sliding function reduce the chattering phenomenon which appeared in the classical discrete sliding mode controller. But the perfor- mance of these tow algorithms is similar. The discrete predictive sliding mode control with the new sliding function reduces the chattering phenomenon with a notable amelioration of the closed loop performance.

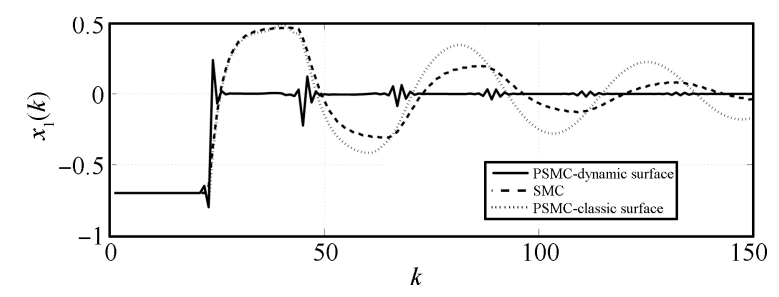

Fig. 1 Evolutions of the state $x_{1}(k)$

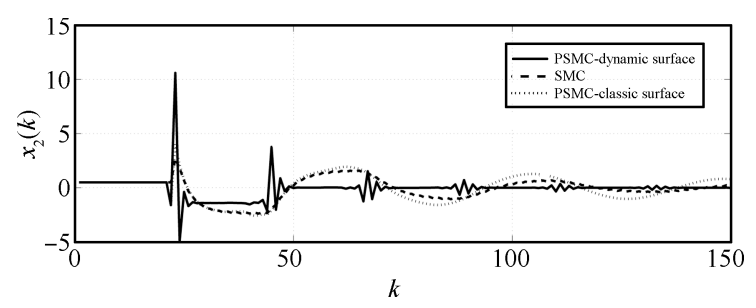

Fig. 2 Evolutions of the state $x_{2}(k)$

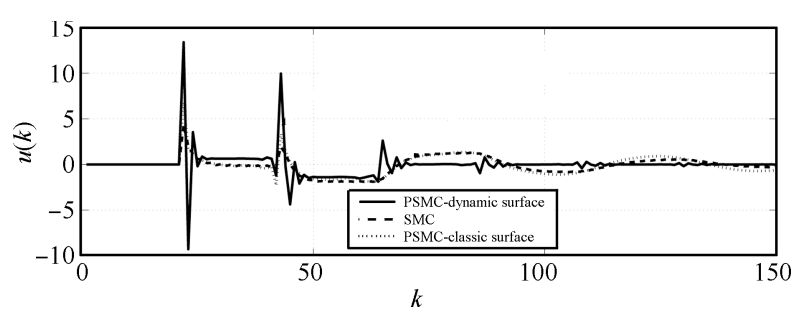

Fig. 3 Evolutions of the control input $u(k)$

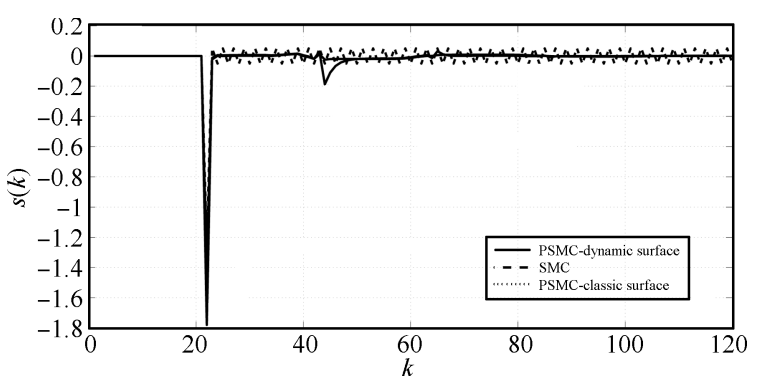

Fig. 4 Evolutions of the sliding function $S(k)$

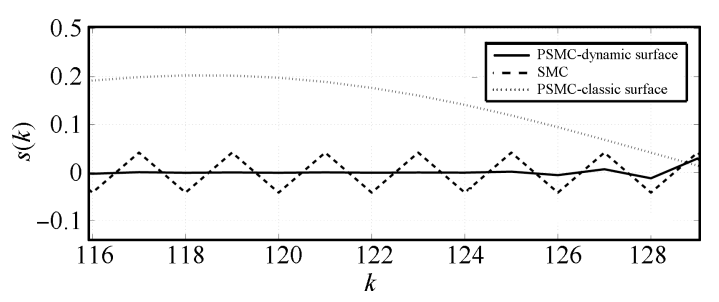

Fig. 5 Zoom of the sliding function $S(k)$

In Fig. 4, the evolutions of the sliding functions are represented and it shows the contribution of the proposed predictive sliding mode control with dynamic sliding function 
in terms of oscillations reduction and convergence speed as shown in Fig. 5.

In the whole, the proposed discrete predictive sliding mode controller with the dynamic sliding function gives a better amelioration than the classical algorithm.

\section{Conclusions}

This paper deals with control, stabilization and chattering problems for discrete-time delay systems. We proposed a new discrete predictive sliding mode control. Using the LMI solvers, a sufficient condition was given to guarantee the existence of a new stable sliding function. The discretetime reaching law was improved by applying this new sliding function and a reference trajectory for discrete-time delay systems.

\section{References}

[1] J. P. Richard. Time-delay systems: An overview of some recent advances and open problems. Automatica, vol.39, no. 10, pp. 1667-1694, 2003.

[2] A. Seuret. Control and Observation of Time-varying Delays: Theory and Application, Ph. D. dissertation, Central School of Lille, France, 2006.

[3] J. E. Normey-Rico, E. F. Camacho. Control of Dead-Time Processes, Berlin: Springer, 2007.

[4] J. P. Richard, F. Gouaisbaut, W. Perruquetti. Sliding mode control in the presence of delay. Kybernetika, vol. 37, no. 3, pp. 277-294, 2001.

[5] Z. L. Liu, J. Zhang, R. Pei. Robust model predictive control of time-delay systems. In Proceedings of IEEE Conference on Control Applications, IEEE, Istanbul, Turkey, vol. 1, pp. 470-473, 2003.

[6] Y. Z. Zhang, M. Liu, W. Jianhui, S. S. Gu, G. Li. Robust model predictive control for uncertain discrete-time system with both states and input delays. In Proceedings of Chinese Control and Decision Conference, IEEE, Yantai, China, pp. 279-284, 2008.

[7] K. Ramakrishnan, G. Ray. Stability criterion with less LMI variables for linear discrete-time systems with additive timedelays. International Journal of Automation and Computing, vol. 8, no. 4, pp. 490-492, 2011.

[8] Y. Y. Shao, X. D. Liu, X. Sun, Z. Su. A new admissibility condition of discrete-time singular systems with time-varying delays. International Journal of Automation and Computing, vol. 9, no, 5, pp. 480-486, 2012.

[9] S. B. Stojanovic, D. L. Debeljkovic. Stability of linear discrete time delay systems: Lyapunov-Krasovskii approach. In Proceedings of the 4th IEEE Conference on Industrial Electronics and Applications, IEEE, Xi'an, China, pp. 24972501, 2009.

[10] M. Yan, Y. Shi. Robust sliding-mode control of uncertain discrete-time systems with time delay. IET Control Theory \& Applications, vol 2, no. 8, pp. 662-674, 2008.

[11] M. X. Yan, Y. W. Jing, Y. G. He. The output feedback variable structure control for discrete-time systems with uncertainties and time delay. In Proceedings of the International Conference on Intelligent Computation Technology and $\mathrm{Au}$ tomation, IEEE, Hunan, China, pp. 516-520, 2008.
[12] H. M. Xiao, W. Y. Chen, C. C. Gao. Discrete sliding-mode control of uncertain systems with time delays. In Proceedings of the International Conference on Modelling, Identification and Control, IEEE, Okayama, Japan, pp. 882-885, 2010.

[13] M. C. Pai. Discrete-time sliding mode control for uncertain systems with state and input delays. International Journal of Systems Science, vol. 41, no. 12, pp. 1501-1510, 2010.

[14] A. Nizar, N. A. Said. A new sliding surface for discrete second order sliding mode control of time delay systems. In Proceedings of the 9th International Multi-Conference on Systems, Signals Devices, IEEE, Chemnitz, Germany, pp. 1-6, 2012 .

[15] A. Nizar, A. S. Nouri. A new sliding surface for discrete second order sliding mode control for time delay systems with time varying uncertainties. International Journal of Computer Applications, vol. 52, no. 21, pp. 16-22, 2012.

[16] V. I. Utkin, H. C. Chang. Sliding mode control on electromechanical systems. Mathematical Problems in Engineering, vol. 8, no. 4-5, pp. 451-473, 2002.

[17] W. Perruquetti, J. P. Barbot. Sliding Mode Control in Engineering, London: Taylor \& Francis, 2002.

[18] E. M. Jafarov. Variable structure control and time-delay systems. A Series of Reference Books and Textbooks, Europe Office, Greece: WSEAS Press, 2009.

[19] A. Bartoszewicz. Discrete-time quasi-sliding-mode control strategies. IEEE Transactions on Industrial Electronics, vol. 45, no. 4, pp.633-637, 1998.

[20] P. Lopez, A. S. Nouri. Elementary theory and practice of control by sliding modes. Mathematics and Applications, SMAI, Springer-Verlag, 2006.

[21] H. Buhler. Regulation by Sliding Mode, Press Lausanne, Polytechnic Romandes Lausanne, 1986.

[22] J. H. Kim, S. H. Oh, D. D. Cho, J. K. Hedrick. Robust discrete-time variable structure control methods. Journal of Dynamic Systems, Measurement, and Control, vol. 122, no. 4 pp. $766-775,2000$.

[23] X. H. Yu, S. H. Yu. Discrete sliding mode control design with invariant sliding sectors. Transactions of the ASME, Journal of Dynamic Systems, Measurement, and Control, vol. 122, no. 4 pp. 776-782, 2000.

[24] C. E. García, D. M. Prett, M. Morari. Model predictive control: Theory and practice - A survey. Automatica, vol. 25, no. 3, pp. 335-348, 1989.

[25] D. W. Clarke, C. Mohtadi, P. S. Tuffs. Generalized predictive control - Part I. The basic algorithm. Automatica, vol. 23, no. 2, pp. 137-148, 1992.

[26] D. W. Clarke, R. Scattolini. Constrained receding-horizon predictive control. IEE Proceedings D: Control Theory and Applications, vol. 138, no. 4, pp. 347-354, 1991.

[27] Z. L. He, M. Wang, S. H. Liu. Discrete sliding mode prediction control of uncertain switched systems. Journal of Systems Engineering and Electronics, vol. 20, no. 5, pp. 10651071, 2009.

[28] O. Camacho. A predictive approach based-sliding mode control. In Proceedings of the 15th Triennial World Congress, IFAC, Barcelona, Spain, 2002. 
[29] W. Garcia-Gabin, D. Zambrano, E. F. Camacho. Sliding mode predictive control of a solar air conditioning plant. Control Engineering Practice, vol.17, no. 6, pp.652-663, 2009.

[30] L. F. Xiao, H. Y. Su, X. Y. Zhang, J. Chu. Discrete variable structure control algorithm for nonlinear systems via sliding mode prediction. In Proceedings of the American Control Conference, IEEE, Minneapolis, Minnesota, USA, pp. 4712$4717,2006$.

[31] M. P. de la Parte, O. Camacho, E. F. Camacho. Development of a GPC-based sliding mode controller. ISA Transactions, vol. 41, no. 1, pp. 19-30, 2002.

[32] H. Ben Mansour, A. Nizar, A. S. Nouri. Predictive sliding mode control for perturbed discrete time delay systems: Robustness analysis. In Proceedings of the International conference on Electrical Engineering and Software Applications, Hammamet, Tunisia, 2013. (Online first)

[33] L. F. Xiao, H. Y. Su, X. Y. Zhang, J. Chu. A new discrete variable structure control algorithm based on sliding mode prediction. In Proceedings of the American Control Conference, IEEE, Portland, OR, USA, vol. 7, pp. 4643-4648, 2005.

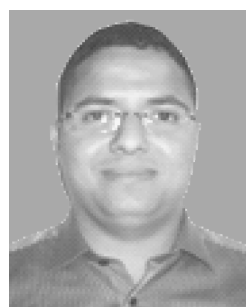

Abdennabi Nizar received the B. Eng. degree in automatic-electrical engineering, and the M. Eng. degree in automatic control from the National School of Engineers of Gabes, Tunisia in 2009 and 2010. Currently, he is a $\mathrm{Ph} . \mathrm{D}$. Candidate at $\mathrm{Nu}-$ merical Control of Industrial Processes, National Engineering School of Gabes, Tunisia. He is currently teaching automatic control in National School of Engineers of

Gabes, Tunisia.

His research interest include discrete sliding mode control, predictive sliding mode control, stability and stabilization analysis of time delay systems.
E-mail: abdennebi.nizar@yahoo.fr (Corresponding author)

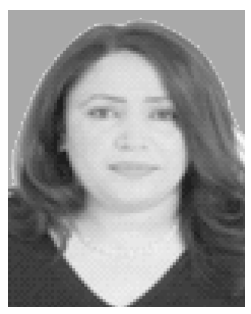

Ben Mansour Houda received her B. Eng. degree in electrical engineering and industrial computing from National School of Engineers of Monastir, Tunisia in 2003 and her M. Eng. degree in automatic and industrial computing from the National School of Engineers of Sfax, Tunisia in 2006. She is now preparing her thesis in electric engineering in National School of engineers of Gabes, Tunisia. She is currently teaching industrial computing and automatic control in High School of Applied Sciences and Technology of Gabes, Tunisia

Her research interests include model predictive control, sliding mode control and predictive sliding mode control for multivariable systems.

E-mail: houda.b.mansour@Gmail.com

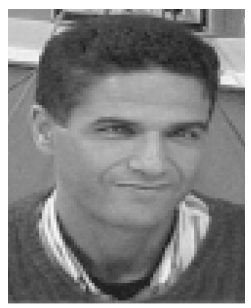

Nouri Ahmed Said received the DEA and the Ph. D. degrees in automatic from National Institute for Applied Sciences, France in 1989 and 1994, respectively. In 2008, he obtained the University Delegation in electric automatic and industrial informatics engineering from National School of Engineers of Sfax, Tunisia. He is professor in automatic control at National School of Engineering of Gabes, Tunisia. He was the chairman of the Electrical Engineering Department of National School of Engineering of Gabes. He is a member of the Research Unit of Numerical Control of Industrial Processes and is the general secretary of the Tunisian Association of Automatic and Numerisation. He is the co-author of the book The Elementary and Practical Theory of the Sliding Mode Control edited by Springer.

His research interests include sliding mode control, numerical control and nonlinear control of industrial processes.

E-mail: ahmedsaid.nouri@enig.rnu.tn 